\title{
Detection of Pulmonary Embolism with Combined Ventilation-Perfusion SPECT and Low-Dose CT: Head-to-Head Comparison with Multidetector CT Angiography
}

\author{
Henrik Gutte ${ }^{1,2}$, Jann Mortensen ${ }^{1}$, Claus Verner Jensen ${ }^{3}$, Camilla Bardram Johnbeck ${ }^{1,2}$, Peter von der Recke ${ }^{1,3}$, \\ Claus Leth Petersen ${ }^{4}$, Jesper Kjærgaard ${ }^{5}$, Ulrik Sloth Kristoffersen ${ }^{1,2}$, and Andreas Kjær ${ }^{1,2}$ \\ ${ }^{I}$ Department of Clinical Physiology, Nuclear Medicine and PET, Rigshospitalet, Copenhagen University Hospital, Copenhagen, \\ Denmark; ${ }^{2}$ Cluster for Molecular Imaging, Faculty of Health Sciences, University of Copenhagen, Copenhagen, Denmark; \\ ${ }^{3}$ Department of Radiology, Rigshospitalet, Copenhagen University Hospital, Copenhagen, Denmark; ${ }^{4}$ Department of Clinical \\ Physiology and Nuclear Medicine, Frederiksberg Hospital, Frederiksberg, Denmark; and ${ }^{5}$ Department of Cardiology, \\ Rigshospitalet, Copenhagen University Hospital, Copenhagen, Denmark
}

\begin{abstract}
The diagnosis of pulmonary embolism (PE) is usually established by a combination of clinical assessment, D-dimer testing, and imaging with either pulmonary ventilation-perfusion (V/Q) scintigraphy or pulmonary multidetector CT (MDCT) angiography. Both V/Q SPECT and MDCT angiography seem to have high diagnostic accuracy. However, only limited data directly comparing these 2 modalities are available. Hybrid $\gamma$-camera/MDCT systems have been introduced and allow simultaneous 3-dimensional lung V/Q SPECT and MDCT angiography, suitable for diagnosing PE. The aim of our study was to compare, in a prospective design, the diagnostic ability of V/Q SPECT, V/Q SPECT combined with low-dose CT, and pulmonary MDCT angiography obtained simultaneously using a combined SPECT/MDCT scanner in patients suspected of having PE. Methods: Consecutive patients from June 2006 to February 2008 suspected of having acute PE were referred to the Department of Nuclear Medicine at Rigshospitalet or Frederiksberg Hospital, Denmark, for V/Q SPECT as a first-line imaging procedure. The number of eligible patients was 196. Patients with positive D-dimer results $(>0.5 \mathrm{mmol} / \mathrm{mL})$ or a clinical assessment with a Wells score greater than 2 were included and underwent V/Q SPECT, lowdose CT, and pulmonary MDCT angiography in a single session. Patient follow-up was 6 mo. Results: A total of 81 simultaneous studies were available for analysis, of which $38 \%$ were from patients with PE. V/Q SPECT had a sensitivity of $97 \%$ and a specificity of $88 \%$. When low-dose CT was added, the sensitivity was still $97 \%$ and the specificity increased to $100 \%$. Perfusion SPECT with low-dose CT had a sensitivity of $93 \%$ and a specificity of $51 \%$. MDCT angiography alone had a sensitivity of $68 \%$ and a specificity of $100 \%$. Conclusion: We conclude that V/Q SPECT in combination with low-dose CT without contrast enhancement has an excellent diagnostic performance and should therefore probably be considered first-line imaging in the workup of PE in most cases.
\end{abstract}

Received Jan. 22, 2009; revision accepted Mar. 23, 2009.

For correspondence or reprints contact: Henrik Gutte, Department of Clinical Physiology, Nuclear Medicine and PET, 4011 Rigshospitalet, Copenhagen University Hospital, Blegdamsvej 3, 2100 Copenhagen, Denmark.

E-mail: henrik.gutte@rh.regionh.dk
Key Words: clinical cardiology; SPECT/CT; CT angiography; V/Q SPECT; pulmonary embolism

J Nucl Med 2009; 50:1987-1992

DOI: 10.2967/jnumed.108.061606

A cute pulmonary embolism (PE) is a severe and potentially fatal disease, with a mortality rate of approximately $30 \%$ if untreated. The incidence is 2 per 1,000 persons per year in Western countries (1-4).

$\mathrm{PE}$ is usually diagnosed through a combination of clinical assessment, blood sampling with D-dimer testing, and imaging with either pulmonary ventilation-perfusion (V/Q) scintigraphy or pulmonary multidetector CT (MDCT) angiography.

Pulmonary CT angiography has been shown to have a higher diagnostic accuracy and specificity than conventional planar V/Q scintigraphy $(5,6)$. Thus, in many institutions, MDCT is first-line imaging in the daily clinical routine for patients suspected of PE (7-9). In addition, MDCT is fast, can yield an alternative diagnosis, and has a high degree of interobserver agreement $(6,7,10,11)$.

Accordingly, in recent years the role of V/Q scintigraphy in the diagnosis of PE has diminished. Among the weaknesses of V/Q scintigraphy, if performed as traditional planar scintigraphy using Prospective Investigation of Pulmonary Embolism Diagnosis (PIOPED) interpretation criteria (12), are high proportions of equivocal studies $(9,12)$ as well as moderate interobserver agreement (6). Yet, the introduction of 3-dimensional V/Q SPECT technology instead of 2-dimensional planar V/Q scintigraphy has in pilot data suggested high diagnostic accuracy for SPECT (13-15). 
Both V/Q SPECT and MDCT angiography appear to have high diagnostic accuracy. However, very limited data directly comparing these two 3-dimensional modalities are available. The only head-to-head comparison currently available found that MDCT was superior in specificity but that V/Q SPECT was superior in sensitivity. However, the study was retrospective, used a 4-slice CT scanner, and allowed up to $3 \mathrm{~d}$ between MDCT and V/Q SPECT scanning (14).

Recently, hybrid $\gamma$-camera/MDCT systems have been introduced. These allow for simultaneous lung V/Q SPECT and MDCT angiography, which may be used for diagnosing PE $(15,16)$. Comparison of simultaneous V/Q SPECT and pulmonary MDCT angiography for the detection of PE has, to our knowledge, never been undertaken.

Therefore, the aim of our study was to assess in a prospective design the diagnostic ability of V/Q SPECT compared with that of pulmonary MDCT angiography performed simultaneously using a dedicated combined SPECT/MDCT scanner in patients suspected of having PE. To do so, we investigated the accuracy of V/Q SPECT and pulmonary MDCT angiography and of combined V/Q SPECT and lowdose pulmonary $\mathrm{CT}$ without contrast agent administration.

\section{MATERIALS AND METHODS}

\section{Study Design, Selection Criteria, and Patients}

The study included consecutive patients suspected of acute PE at Rigshospitalet (third-line referral hospital) or Frederiksberg Hospital (primary referral hospital), Copenhagen, Denmark, from June 2006 to February 2008. All patients were referred to the Department of Nuclear Medicine at Rigshospitalet or Frederiksberg Hospital, Denmark, for V/Q SPECT as a first-line imaging procedure. The Local Scientific Ethics committee approved the study (H-KF-308395). Written informed consent was obtained from all patients. The study protocol complied with the guidelines of the Helsinki Declaration on human experimentation. Patients were eligible if there was suspicion of PE, defined as an acute onset of new-or a worsening of-shortness of breath or chest pain without any obvious cause, combined with positive D-dimer results $(>0.5 \mathrm{mmol} / \mathrm{mL})$ or a clinical assessment with a Wells score (17) greater than 2.

Among 196 consecutive patients referred to our departments, 96 patients $(51 \mathrm{men})$ were excluded from the study because of contraindications to CT: an allergy to iodine contrast agents (2 patients); impaired renal function, defined as a P-creatinine level greater than $0.120 \mathrm{mmol} / \mathrm{L}$ (46 patients); and women under the age of $40 \mathrm{y}$ (14 patients). Patients were also excluded from the study if they decided not to participate (6 patients), were not able to cooperate (11 patients), or could not have a peripheral venous access established ( 3 patients) or if there were hardware or software issues (8 patients). Three additional patients were excluded for other reasons, and 3 patients were ineligible to participate because of low clinical suspicion due to a Wells score of less than 2 and a negative $\mathrm{D}$-dimer result.

All patients consented to diagnostic testing, including pulmonary MDCT angiography, low-dose CT without contrast enhancement, V/Q SPECT, blood pressure measurement, and blood sampling.

All patients had at least 6 mo of follow-up after the scans, with telephone interviews and review of hospital files.
Pulmonary MDCT angiography, low-dose CT, and V/Q SPECT were performed in the same session using a Precedence scanner (Philips), which is an integrated 2-head $\gamma$-camera combined with an MDCT scanner (16-slice Brilliance).

\section{CT Acquisition Parameters and Image Reconstruction}

The CT protocol consisted of 2 successive acquisitions with the patients scanned supine. The patients were allowed to breathe freely between the 2 scans. The first acquisition was a low-dose CT scan $(140 \mathrm{kV}, 20 \mathrm{mAs} / \mathrm{slice}, 16 \times 1.5$ collimator, 0.5 -s rotation time, and pitch of 0.813 ) that was used for attenuation correction of V/Q SPECT data and obtained during tidal breathing.

The second acquisition was pulmonary MDCT angiography during a deep-inspiration breath-hold $(120 \mathrm{kV}, 230 \mathrm{mAs} / \mathrm{slice}$, $16 \times 0.75 \mathrm{~mm}$ collimator, 0.5 -s rotation time, and pitch of 0.94 ). The images were acquired in the cephalocaudal direction and in a $512 \times 512$ matrix.

Biphasic administration of contrast medium (350 mg of iodine/ $\mathrm{mL}$, Optiray; Tyco Healthcare/Mallinckrodt) was performed with an automatic injection pump (Optivantage; Tyco Healthcare/ Mallinckrodt). The first phase consisted of administration of $80 \mathrm{~mL}$ at $4.3 \mathrm{~mL} / \mathrm{s}$ into a cubital vein followed by a saline chaser bolus of $45 \mathrm{~mL}$ injected at a flow rate of $4.3 \mathrm{~mL} / \mathrm{s}$.

To ensure optimal opacification of the lung arteries, we obtained the scan using bolus tracking, entering a circular region of interest in the trunk of the pulmonary artery. The threshold for triggering was preset at 100 Hounsfield units.

The pulmonary MDCT angiographic scans were reconstructed with a contiguous slice thickness of $0.8 \mathrm{~mm}$ and were reviewed on a dedicated workstation (Extended Brilliance Workspace, version 3.0 ; Philips).

All angiographic scans were evaluated by 2 readers (with 15 and $8 \mathrm{y}$ of experience in evaluating pulmonary MDCT angiography) who were unaware of the clinical history. The diagnosis of PE was based on failure to enhance the entire lumen because of a filling defect (18).

\section{Pulmonary V/Q SPECT Acquisition and Data Reconstruction}

Pulmonary SPECT included a perfusion SPECT study and ventilation SPECT study obtained simultaneously within $13 \mathrm{~min}$ (i.e., 36 projections of $20 \mathrm{~s}$ each per head over $180^{\circ}$ ) immediately after the MDCT acquisition with the patient supine. Perfusion studies were performed after intravenous injection of $150 \mathrm{MBq}$ of ${ }^{99} \mathrm{~m}$ Tc-macroaggregated albumin (Mallinckrodt) during 2 respiratory cycles. Ventilation scintigraphy was conducted during quiet tidal inhalation of ${ }^{81 \mathrm{~m}} \mathrm{Kr}$ made at our institution, which was extracted from a $600-\mathrm{MBq}{ }^{81} \mathrm{Rb}-{ }^{81 \mathrm{~m}} \mathrm{Kr}$ generator by oxygen flow of $1 \mathrm{~L} \cdot \mathrm{min}^{-1}$. Both studies were performed simultaneously with low-energy general-purpose collimators and acquired in a $128 \times$ 128 matrix.

SPECT datasets were corrected for attenuation using the lowdose CT acquisition (without contrast medium) with iterative reconstruction using the software Autospect + and Astonish with 3 iterations and 16 subsets (Philips).

The V/Q SPECT images were interpreted on a Jetstream workstation (Philips) by 2 readers (with 15 and 2 y of experience) who were unaware of the clinical history of the patients. The size and segmental location of all perfusion and ventilation defects were noted. PE was diagnosed if one or more mismatched perfusion defects with normal ventilation were present. V/Q 
SPECT datasets were first reviewed independently, and a diagnosis was established. Then, they were viewed in combination and fused with the pulmonary low-dose CT, and a new diagnosis was obtained. In a new reading, the perfusion SPECT was reviewed in combination with the low-dose CT, and a new diagnosis was established. We used an automatically 3-dimensional imaging registration software tool that displayed the low-dose CT, V/Q SPECT, and fusion images in axial, coronal, and sagittal planes (Syntegra, version 2.3.1; Philips).

The final diagnosis was made at a side-by-side consensus reading of all lesions detected on pulmonary MDCT angiography and V/Q SPECT using all available information: electrocardiography, transthoracic echocardiography, Doppler sonography of the lower-extremity veins, D-dimer levels, clinical data, and follow-up from hospital files or telephone interviews. PE was ruled out if patients had no evidence of PE in the clinical data or follow-up (including clinical time course), if they responded to treatment within 6 mo, or if they died and PE was an unlikely cause of death.

We did not usually repeat the V/Q SPECT or MDCT angiography, unless requested by the clinicians.

\section{Statistical Analysis}

Diagnostic performance was calculated as sensitivity, specificity, positive predictive value, negative predictive value, and accuracy. Ninety-five percent confidence intervals (19) were calculated. Patient characteristics were compared between the no-PE and PE groups using a 2-sample $t$ test. D-dimer was $\log 10$-transformed to obtain a gaussian distribution. $P$ values of less than 0.05 were considered significant. All statistical analyses were performed using SPSS software (version 15.0; SPSS Inc.) for Windows (Microsoft).

\section{RESULTS}

During the 18-mo recruiting period, 196 patients were screened and 100 were eligible for the study. Of the 100 patients eligible for the study, 6 were excluded because of poor contrast enhancement in the pulmonary vessels, and 8 were excluded because poor technical quality rendered the V/Q SPECT images uninterpretable.

For 5 patients, no final diagnosis could be determined (i.e., no reference was available), leaving a total of 81 patients available for analysis of the diagnostic performance of the imaging modalities. For V/Q SPECT alone, 4 of the 81 cases were inconclusive, leading to a nondiagnostic rate of $5 \%$. For perfusion SPECT with low-dose CT, we found a nondiagnostic rate of $17 \%$. In all other cases, both the pulmonary MDCT angiography and the V/Q SPECT in combination with low-dose CT could be interpreted as positive or negative, that is, a diagnostic rate of $100 \%$. Patient characteristics are shown in Table 1.

V/Q SPECT alone had a sensitivity of $97 \%$ and a specificity of $88 \%$. When low-dose CT was added, the sensitivity was still $97 \%$ and the specificity increased to $100 \%$. MDCT angiography alone had a sensitivity of $68 \%$ and a specificity of $100 \%$. Perfusion SPECT with low-dose CT had a sensitivity of $93 \%$ and a specificity of $51 \%$.

Further details on diagnostic performance are given in Tables 2 and 3.

\begin{tabular}{|c|c|c|c|}
\hline Characteristic & $\begin{array}{l}\text { No PE } \\
(n=50)\end{array}$ & $\begin{array}{c}\text { PE } \\
(n=31)\end{array}$ & $P$ \\
\hline Age $(y)$ & $68 \pm 12$ & $63 \pm 14$ & 0.14 \\
\hline Height $(\mathrm{cm})$ & $171 \pm 11$ & $175 \pm 9$ & 0.33 \\
\hline Weight (kg) & $74 \pm 18.2$ & $81 \pm 23.5$ & 0.18 \\
\hline $\begin{array}{l}\text { Body mass index } \\
\left(\mathrm{kg} / \mathrm{m}^{2}\right)\end{array}$ & $25.1 \pm 5.6$ & $26.5 \pm 6.6$ & 0.30 \\
\hline $\begin{array}{l}\text { Systolic blood } \\
\text { pressure }(\mathrm{mm} \mathrm{Hg})\end{array}$ & $138 \pm 20$ & $133 \pm 17$ & 0.31 \\
\hline $\begin{array}{l}\text { Diastolic blood } \\
\text { pressure mm Hg) }\end{array}$ & $78 \pm 15$ & $82 \pm 13$ & 0.57 \\
\hline Pulse (bpm) & $82 \pm 18$ & $87 \pm 14$ & 0.88 \\
\hline Wells score & $2.2 \pm 1.8$ & $3.1 \pm 2.4$ & 0.05 \\
\hline D-dimer & $2.0 \pm 2.0$ & $5.0 \pm 4.8$ & $>0.0001^{*}$ \\
\hline
\end{tabular}

The supplemental table lists patients with discrepancies (supplemental materials are available online only at http:// jnm.snmjournals.org). In 10 patients with a PE diagnosis established at the consensus reading, PE could not be visualized on MDCT angiography. In 4 of these patients, PE could be localized when the pulmonary MDCT angiography datasets were combined with the V/Q SPECT. In all 4 patients, embolic thrombi were visualized in the same regions as the mismatched defects seen on V/Q SPECT; in 2 of these patients, the emboli were located in small subsegmental arteries. One patient with myelomatosis and a D-dimer of 8.7 had a negative PE diagnosis on V/Q SPECT, and MDCT angiography demonstrated a relatively large embolus in the left upper lobe at arterial level 2. Ultrasonography confirmed DVT of the lower-extremity vein. In the remaining 5 patients with PE on V/Q SPECT combined with low-dose CT, the pulmonary MDCT angiography acquisitions were technically perfect, with opacification of the pulmonary arteries at arterial levels 5-6.

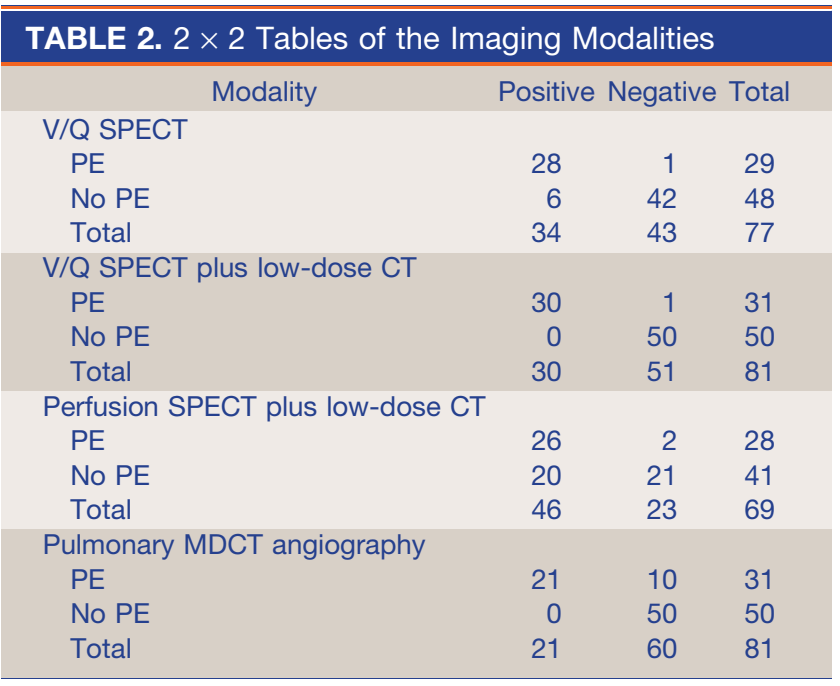




\begin{tabular}{|c|c|}
\hline Modality & Performance \\
\hline \multicolumn{2}{|l|}{ V/Q SPECT } \\
\hline Sensitivity (\%) & $97(82-100)$ \\
\hline Specificity (\%) & $88(75-95)$ \\
\hline PPV (\%) & $82(65-93)$ \\
\hline NPV (\%) & $98(88-100)$ \\
\hline Accuracy (\%) & $91(83-93)$ \\
\hline Nondiagnostic rate (\%) & $5(1-12)$ \\
\hline \multicolumn{2}{|c|}{ V/Q SPECT plus low-dose CT } \\
\hline Sensitivity (\%) & 97 (83-99) \\
\hline Specificity (\%) & $100(93-100)$ \\
\hline PPV (\%) & $100(88-100)$ \\
\hline NPV (\%) & $98(90-100)$ \\
\hline Accuracy (\%) & $99(93-100)$ \\
\hline Nondiagnostic rate (\%) & $0(0-4)$ \\
\hline \multicolumn{2}{|c|}{ Perfusion SPECT plus low-dose CT } \\
\hline Sensitivity (\%) & $93(81-98)$ \\
\hline Specificity (\%) & $51(43-55)$ \\
\hline PPV (\%) & $57(49-60)$ \\
\hline NPV (\%) & $91(76-98)$ \\
\hline Accuracy (\%) & $68(58-72)$ \\
\hline Nondiagnostic rate (\%) & $17(10-28)$ \\
\hline \multicolumn{2}{|c|}{ Pulmonary MDCT angiography } \\
\hline Sensitivity (\%) & 68 (49-83) \\
\hline Specificity (\%) & $100(93-100)$ \\
\hline PPV (\%) & $100(84-100)$ \\
\hline NPV (\%) & $83(71-92)$ \\
\hline Accuracy (\%) & $88(78-94)$ \\
\hline Nondiagnostic rate (\%) & $0(0-4)$ \\
\hline \multicolumn{2}{|c|}{$\begin{array}{l}\text { PPV = positive predictive value; NPV = negative predictive } \\
\text { value. } \\
\text { Values in parenthesis are } 95 \% \text { confidence intervals. }\end{array}$} \\
\hline
\end{tabular}

When the V/Q SPECT datasets alone were assessed, 6 patients had a false-positive diagnosis of PE: 3 patients had mismatched defects on the V/Q SPECT scans because of interlobar fissures, and 3 patients had chronic obstructive pulmonary disease and had mismatched defects because of paraseptal emphysema, pneumonic infiltration, atelectasis, and pleural fluid as seen on low-dose CT (Figs. 1 and 2).

\section{DISCUSSION}

In the present study, we investigated prospectively the diagnostic performance of V/Q SPECT and pulmonary MDCT angiography alone and V/Q SPECT in combination with low-dose pulmonary CT in consecutive patients suspected of having PE. Our major findings were that V/Q SPECT alone and V/Q SPECT combined with low-dose CT had a high sensitivity, and it was higher than that of pulmonary MDCT angiography. In addition, pulmonary MDCT angiography and V/Q SPECT in combination with low-dose CT had a high specificity, whereas V/Q SPECT alone had a lower specificity. Furthermore, we demonstrated that perfusion SPECT combined with low-dose CT had a high sensitivity and a low specificity.
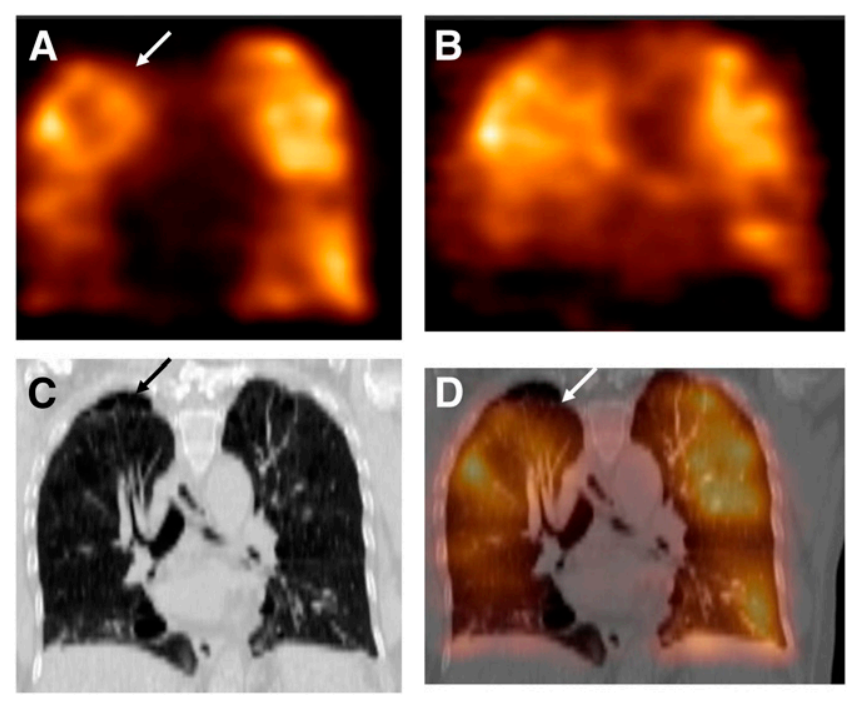

FIGURE 1. (A) Coronal perfusion SPECT revealed a moderate mismatched defect (arrow) in apex of right lung and some matched defects in both lungs. (B) Ventilation SPECT showed inhomogeneous activity in right apex and several matched defects and reversed mismatched defects (ventilation defects but normal perfusion). (C) Coronal low-dose CT revealed emphysema, and in apically mismatched area several bullae (arrow) were demonstrated. (D) Bullae and mismatched defect (arrow) in right apex corresponded on fused perfusion SPECT and low-dose CT.

To our knowledge, this was the first prospective study on simultaneously obtained V/Q SPECT and pulmonary CT angiography and also the first study using a dedicated hybrid SPECT/MDCT scanner comparing the diagnostic performance of V/Q SPECT, pulmonary MDCT angiography, and V/Q SPECT combined with low-dose CT on a head-to-head basis in patients suspected of having PE.

With regard to sensitivity, specificity, and accuracy, we found good agreement with a previous retrospective study (14), which found values of $97 \%$, 91\%, and 94\%, respectively, for V/Q SPECT and values of $86 \%, 98 \%, 93 \%$, respectively, for MDCT. We found a sensitivity, specificity, and accuracy of $97 \%, 88 \%$, and 91\%, respectively, for V/Q SPECT; 68\%, 100\%, and 88\%, respectively, for MDCT; and $97 \%, 100 \%$, and 99\%, respectively, for V/Q SPECT/ low-dose CT. The PIOPED II study of the diagnostic accuracy of pulmonary MDCT angiography used a composite reference standard to establish a PE diagnosis. The reference standard included findings on V/Q lung scans (planar imaging), conventional digital subtraction angiograms, and venous sonography. The sensitivity found for pulmonary MDCT angiography was $83 \%$ (7). We had a slightly lower sensitivity of $68 \%$ for MDCT. We have no obvious explanation for the difference in sensitivity, but the differences in study populations and algorithms for final diagnosis may have been contributing factors.

To our knowledge, this was the first time perfusion SPECT with low-dose CT has been evaluated in patients with PE, and 

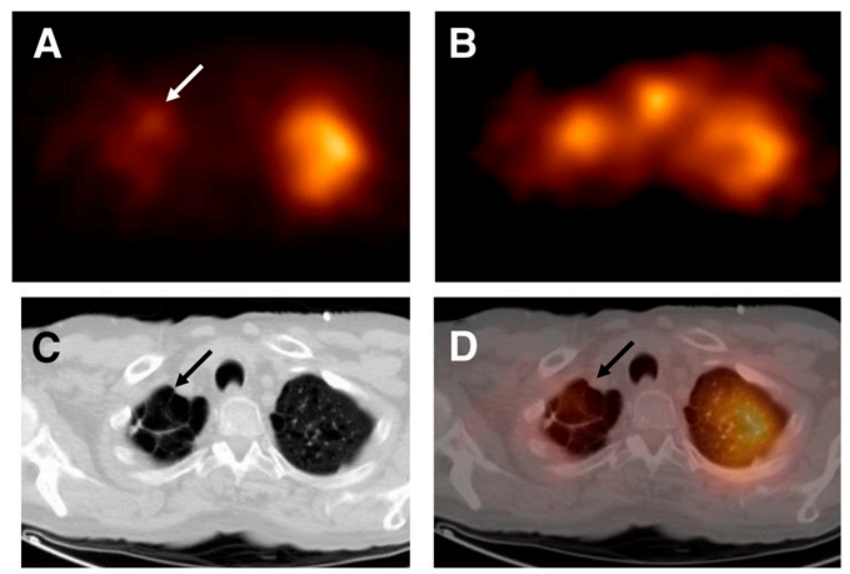

FIGURE 2. (A) Axial perfusion SPECT revealed moderate mismatched defect (arrow) with decreased perfusion in right apex but normal perfusion in left apex. (B) Ventilation SPECT showed higher degree of ventilation in both apices but ventilation defect in left apex (reversed mismatch). (C) Lowdose CT revealed emphysema and several bullae (arrow) in right apex. (D) Bullae and mismatched defect (arrow) corresponded on fused perfusion SPECT and low-dose CT.

we demonstrated a high sensitivity but a significantly lower specificity. However, when ventilation SPECT was added, a significantly higher specificity and diagnostic rate were found that were similar to those of pulmonary MDCT angiography. Therefore, interpretation should be performed in the context of ventilation SPECT and not just with low-dose $\mathrm{CT}$ to avoid a high number of false-positive diagnostic results.

We reported all mismatched defects on SPECT as PE. Alternative reasons (14) for mismatched V/Q defects include a few, rare nonembolic diseases such as pulmonary vasculitis; extrinsic vascular compression such as vessel stenosis, lung cancer, and nodal enlargement; and therapeutic interventions such as radiation therapy $(20,21)$. However, because we could exclude such a differential diagnosis by follow-up, anamnesis, and blood sampling and, most important, by findings on head-to-head comparisons on pulmonary MDCT angiography, we further reduced the risk of an incorrect conclusion.

Taken together, we found that V/Q SPECT in combination with pulmonary low-dose CT seems useful as a first-line routine test for PE, with excellent diagnostic performance.

At present, many centers use only pulmonary MDCT, but this might be suboptimal because of its possible lower sensitivity and higher radiation dose. Reasons for extensive use of MDCT may include its round-the-clock availability, its lower cost, its high frequency of conclusive results, and inexperience with V/Q SPECT. Recently proposed algorithms for imaging evaluation of patients suspected of having PE have omitted V/Q scintigraphy from the work-up (22), and some guidelines considered V/Q scintigraphy only as an alternative when patients cannot undergo MDCT because of severe renal insufficiency or allergy to intravenous contrast agents or when a CT-based strategy is inconclusive $(23,24)$. A total of 46 patients (24\%) were excluded from the present study because of renal dysfunction, indicating that MDCT may not be an option in many patients. This is an important strength of a scintigraphic approach.

A high frequency of equivocal tests $(\leq 73 \%$ of all examinations in some studies (12)) previously hampered lung scintigraphy using the planar technique for diagnosing PE. Using SPECT in V/Q scintigraphy has markedly reduced the frequency of equivocal tests (25-27).

Accordingly, in our study only 4 patients (5\%) had inconclusive V/Q SPECT findings. However, on MDCT and V/Q SPECT plus low-dose CT, no findings were inconclusive.

In contrast to MDCT angiography, V/Q SPECT is a functional technique, as it does not identify the embolus itself but rather its physiologic consequence, the perfusion defect. The addition of low-dose CT without contrast agent to V/Q SPECT increases confidence in the reading while reducing inconclusive studies from 5\% with SPECT alone to $0 \%$ with SPECT combined with low-dose CT. In addition, specificity improves, with fewer false-positive interpretations (from 18\% to $0 \%$ ). This improvement is mainly due to low-dose-CT findings that give alternative explanations for subtle perfusion defects that otherwise would have been interpreted as PE on SPECT alone.

In the interpretation criteria of both the original PIOPED study and the Prospective Investigative Study of Acute Pulmonary Embolism Diagnosis, chest radiography was part of the assessment algorithm in diagnosing PE $(12,28)$. Low-dose CT abolishes the need for chest radiography and increases sensitivity, specificity, and accuracy, compared with radiography, because of increased visualization of atelectasis, emphysema, and other lesions that might cause perfusion and ventilation defects on lung scintigraphy. Thus, the addition of low-dose CT to SPECT gives the same possibility of yielding an alternative diagnosis, as is the case with MDCT angiography. Although inherently inferior to diagnostic CT with a contrast agent, low-dose CT without a contrast agent provided satisfactorily relevant diagnostic information to determine the origin of $\mathrm{V} / \mathrm{Q}$ SPECT lesions. When V/Q SPECT datasets alone were assessed, 6 patients had a false-positive diagnosis of $\mathrm{PE}, 3$ patients had mismatched defects on the V/Q SPECT scans because of interlobar fissures, and 3 patients with chronic obstructive pulmonary disease had mismatched defects because of paraseptal emphysema, pneumonic infiltration, atelectasis, and pleural fluid. All these lesions were seen on low-dose CT. Our procedure requires a combined SPECT/ CT scanner, and the addition of low-dose CT increases the scan time by only $1 \mathrm{~min}$. Low-dose CT adds only $1 \mathrm{mSv}$ to the radiation dose of $2.0 \mathrm{mSv}$ from SPECT (29), comparing favorably with the $11 \mathrm{mSv}$ (30) of pulmonary MDCT angiography.

V/Q SPECT combined with low-dose CT could easily be applied as a routine method. Immobilization during V/Q 
SPECT combined with low-dose CT lasted 20 min and was well tolerated even by critically ill patients. Only 11 of 196 were ineligible to participate because of discomfort, shortness of breath, or lack of cooperation in the supine position. Because we performed pulmonary MDCT angiography and cardiac MDCT angiography in addition to performing V/Q SPECT and low-dose CT, the patients were placed in the scanner for approximately $45 \mathrm{~min}$ - longer than if only a V/Q SPECT had been performed. Our experience is that only $2 \%-3 \%$ are not able to cooperate with V/Q SPECT, which is our daily routine procedure.

Because we excluded patients who had contraindications to MDCT angiography, the conclusions of the study apply strictly to patients who could not safely undergo MDCT angiography. Thus, whether the same results would be obtained in patients who cannot undergo MDCT angiography is unknown.

\section{CONCLUSION}

With combined scanners, lung scintigraphy performed as V/Q SPECT in combination with low-dose CT without contrast enhancement has excellent diagnostic performance and should probably be considered the first-line imaging test in the work-up of PE in most cases.

\section{ACKNOWLEDGMENTS}

We are grateful to the technologists and radiographers at the Departments of Clinical Physiology and Nuclear Medicine, Frederiksberg Hospital, and at the Departments of Clinical Physiology, Nuclear Medicine, and PET, Rigshospitalet, for invaluable assistance.

\section{REFERENCES}

1. Anderson FA Jr, Wheeler HB, Goldberg RJ, et al. A population-based perspective of the hospital incidence and case-fatality rates of deep vein thrombosis and pulmonary embolism. The Worcester DVT Study. Arch Intern Med. 1991;151:933-938.

2. Hansson PO, Welin L, Tibblin G, Eriksson H. Deep vein thrombosis and pulmonary embolism in the general population. 'The Study of Men Born in 1913.' Arch Intern Med. 1997;157:1665-1670.

3. Silverstein MD, Heit JA, Mohr DN, Petterson TM, O'Fallon WM, Melton LJ III. Trends in the incidence of deep vein thrombosis and pulmonary embolism: a 25year population-based study. Arch Intern Med. 1998;158:585-593.

4. Oger E. Incidence of venous thromboembolism: a community-based study in Western France. EPI-GETBP Study Group. Groupe d'Etude de la Thrombose de Bretagne Occidentale. Thromb Haemost. 2000;83:657-660.

5. Grenier PA, Beigelman C. Spiral computed tomographic scanning and magnetic resonance angiography for the diagnosis of pulmonary embolism. Thorax. 1998;53(suppl):25S-31S

6. Blachere H, Latrabe V, Montaudon M, et al. Pulmonary embolism revealed on helical CT angiography: comparison with ventilation-perfusion radionuclide lung scanning. AJR. 2000;174:1041-1047.

7. Stein PD, Fowler SE, Goodman LR, et al. Multidetector computed tomography for acute pulmonary embolism. N Engl J Med. 2006;354:2317-2327.
8. Stein PD, Woodard PK, Weg JG, et al. Diagnostic pathways in acute pulmonary embolism: recommendations of the PIOPED II investigators. Radiology. 2007;242:15-21.

9. Strashun AM. A reduced role of V/Q scintigraphy in the diagnosis of acute pulmonary embolism. J Nucl Med. 2007;48:1405-1407.

10. Mayo JR, Remy-Jardin M, Muller NL, et al. Pulmonary embolism: prospective comparison of spiral CT with ventilation-perfusion scintigraphy. Radiology. 1997;205:447-452.

11. Coche E, Verschuren F, Keyeux A, et al. Diagnosis of acute pulmonary embolism in outpatients: comparison of thin-collimation multi-detector row spiral CT and planar ventilation-perfusion scintigraphy. Radiology. 2003;229: 757-765.

12. PIOPED Investigators. Value of the ventilation/perfusion scan in acute pulmonary embolism: results of the prospective investigation of pulmonary embolism diagnosis (PIOPED). The PIOPED Investigators. JAMA. 1990;263: 2753-2759.

13. Bajc M, Bitzén U, Olsson B, Perez de Sá V, Palmer J, Jonson B. Lung ventilation/perfusion SPECT in the artificially embolized pig. J Nucl Med. 2002;43:640-647.

14. Reinartz P, Wildberger JE, Schaefer W, Nowak B, Mahnken AH, Buell U. Tomographic imaging in the diagnosis of pulmonary embolism: a comparison between V/Q lung scintigraphy in SPECT technique and multislice spiral CT. J Nucl Med. 2004;45:1501-1508.

15. Gutte H, Mortensen J, Jensen C, et al. Added value of combined simultaneous lung ventilation-perfusion single-photon emission computed tomography/multislice-computed tomography angiography in two patients suspected of having acute pulmonary embolism. Clin Resp J. 2008;1:52-55.

16. Bailey DL, Roach P, Bailey E, Hewlett J, Keijzers R. Development of a costeffective modular SPECT/CT scanner. Eur J Nucl Med Mol Imaging. 2007; 34:1415-1426.

17. Wells PS, Ginsberg JS, Anderson DR, et al. Use of a clinical model for safe management of patients with suspected pulmonary embolism. Ann Intern Med. 1998;129:997-1005.

18. Lake DR, Kavanagh JJ, Ravenel JG, Schoepf UJ, Costello P. Computed tomography and pulmonary embolus: a review. Semin Ultrasound CT MR. 2005;26:270-280.

19. Clopper CJ, Pearson ES. The use of confidence or fiducial limits illustrated in the case of the binomial. Biometrika. 1934;26:404-413.

20. Beharry N, Coulden R, Peters M. Pulmonary embolism. In: Ell P, Gambhir S, eds. Nuclear Medicine in Clinical Diagnosis and Treatment. Edinburgh, U.K.: Churchill Livingstone; 2004:883-897.

21. De Geeter FW, Reinartz P, Buell U. Tomographic imaging in the diagnosis of pulmonary embolism: still, we do not know. J Nucl Med. 2005;46:21192121.

22. British Thoracic Society guidelines for the management of suspected acute pulmonary embolism. Thorax. 2003;58:470-483.

23. Kyrle PA, Eichinger S. New diagnostic strategies for pulmonary embolism. Lancet. 2008;371:1312-1315.

24. Remy-Jardin M, Pistolesi M, Goodman LR, et al. Management of suspected acute pulmonary embolism in the era of CT angiography: a statement from the Fleischner Society. Radiology. 2007;245:315-329.

25. Leblanc M, Leveillee F, Turcotte E. Prospective evaluation of the negative predictive value of V/Q SPECT using ${ }^{99 \mathrm{~m} T c-T e c h n e g a s . ~ N u c l ~ M e d ~ C o m m u n . ~}$ 2007;28:667-672.

26. Bajc M, Olsson CG, Olsson B, Palmer J, Jonson B. Diagnostic evaluation of planar and tomographic ventilation/perfusion lung images in patients with suspected pulmonary emboli. Clin Physiol Funct Imaging. 2004;24:249-256.

27. Bajc M, Olsson B, Palmer J, Jonson B. Ventilation/perfusion SPECT for diagnostics of pulmonary embolism in clinical practice. J Intern Med. 2008; 264:379-387.

28. Miniati M, Pistolesi M, Marini C, et al. Value of perfusion lung scan in the diagnosis of pulmonary embolism: results of the Prospective Investigative Study of Acute Pulmonary Embolism Diagnosis (PISA-PED). Am J Respir Crit Care Med. 1996;154:1387-1393.

29. Radiation dose to patients from radiopharmaceuticals (addendum 2 to ICRP publication 53). Ann ICRP. 1998;28:1-126.

30. ImPACT CT patient dosimetry calculator (version 1.0). Available at: http://www.impactscan.org/ctdosimetry.htm. Accessed September 22, 2009. 